\title{
On the Equivalence of B-Rigidity and C-Rigidity for Quasitoric Manifolds
}

\author{
Jin Hong Kim \\ Department of Mathematics Education, Chosun University, 309 Pilmundaero, Dong-gu, Gwangju 501-759, Republic of Korea
}

Correspondence should be addressed to Jin Hong Kim; jinhkim11@gmail.com

Received 26 April 2014; Accepted 4 June 2014; Published 15 June 2014

Academic Editor: Christian Corda

Copyright (C) 2014 Jin Hong Kim. This is an open access article distributed under the Creative Commons Attribution License, which permits unrestricted use, distribution, and reproduction in any medium, provided the original work is properly cited.

\begin{abstract}
For quasitoric manifolds and moment-angle complexes which are central objects recently much studied in toric topology, there are several important notions of rigidity formulated in terms of cohomology rings. The aim of this paper is to show that, among other things, Buchstaber-rigidity (or B-rigidity) is equivalent to cohomological-rigidity (or C-rigidity) for simple convex polytopes supporting quasitoric manifolds.
\end{abstract}

\section{Introduction and Main Results}

In general, a cohomology ring of a given manifold is not enough to determine the manifold completely. However, there are some cases where we can characterize a given manifold in terms of a cohomology ring and which have recently attracted a great amount of attention in toric topology (see $[1,2])$. For example, certain Bott manifolds and quasitoric manifolds, but not all of them, are such cases. The aim of this paper is, roughly speaking, to establish certain equivalence between two well-known notions of rigidity which essentially characterize quasitoric manifolds and also are formulated in terms of cohomology rings.

In order to describe our results more precisely, we first need to collect some definitions and notations. To do so, throughout this paper $\mathbf{k}$ will denote a field of characteristic zero. A quasitoric manifold $M$ of dimension $2 n$ is a closed $2 n$ dimensional smooth manifold with a locally standard action of an $n$-torus $T^{n}:=\left(S^{1}\right)^{n}$ whose orbit space is a simple convex polytope $P$. The combinatorial structure of $P$ can be decoded from the equivariant cohomology ring $H_{T^{n}}^{*}(M ; \mathbf{k})$ of $M$. The reason is that the equivariant cohomology ring of $M$ is isomorphic to the Stanley-Reisner face $\operatorname{ring} \mathbf{k}(K)$ of the dual $K=(\partial P)^{*}$ of the boundary $\partial P$ of $P$ and that the StanleyReisner face ring $\mathbf{k}(K)$ is in turn obtained by using certain combinatorial information of $P$ (refer to, e.g., [2], Theorem 4.8). In a similar vein, it is also expected that one can possibly obtain some information on a simple convex polytope $P$ from the usual cohomology ring of the manifold $M$. If we have a quasitoric manifold $M$ over a simple convex polytope $P$, from now on we will say that $P\left(\right.$ or $\left.K=(\partial P)^{*}\right)$ supports the quasitoric manifold $M$, for simplicity.

From these contexts, it is natural to give Definition 1. In order to explain it, recall first that the faces of a convex polytope form a face poset (or face lattice) where the partial ordering is by set containment of faces. Two polytopes are defined to be combinatorially isomorphic or combinatorially equivalent if their face posets are isomorphic (refer to [1], Section 1.1). An analogous definition obviously applies to two simplicial complexes.

Definition 1. A simple convex polytope $P$ is said to be cohomologically rigid, or simply C-rigid, if the following two conditions hold.

(i) There exists a quasitoric manifold $M$ over $P$.

(ii) Let $M^{\prime}$ be another quasitoric manifold over a simple convex polytope $P^{\prime}$ such that as a ring

$$
H^{*}(M ; \mathbf{k}) \cong H^{*}\left(M^{\prime} ; \mathbf{k}\right) .
$$

Then $P^{\prime}$ is combinatorially equivalent to $P$.

As mentioned above, the Stanley-Reisner face ring $\mathbf{k}(K)$ contains certain significant information of a simple convex 
polytope $P$ supporting the quasitoric manifold, and the dimension of its Tor algebra gives rise to the bigraded Betti numbers $\beta_{i, 2 j}(P)$ which are purely combinatorial invariants of the polytope. It can be shown that the cohomology ring of the moment-angle complex $\mathscr{Z}_{K}$ of the simplicial complex $K=$ $(\partial P)^{*}$ is isomorphic to $\operatorname{Tor}(\mathbf{k}(K) ; \mathbf{k}$ ) (refer to [1], Theorem 7.6, and see Section 2 for the definition of a moment-angle complex $\mathscr{Z}_{K}$ ). Hence, it will be also natural to consider the following notion of rigidity, introduced first by Buchstaber in [3].

Definition 2. A simplicial complex $K$ is said to be Buchstaberrigid, or simply $B$-rigid, if the following condition holds.

(i) Let $K^{\prime}$ be another simplicial complex such that as a ring

$$
H^{*}\left(\mathscr{Z}_{K} ; \mathbf{k}\right) \cong H^{*}\left(\mathscr{Z}_{K^{\prime}} ; \mathbf{k}\right)
$$

Then $K^{\prime}$ is combinatorially equivalent to $K$.

Note that there are simple convex polytopes $P$ supporting quasitoric manifolds which are not C-rigid such that their dual simplicial complexes $K=(\partial P)^{*}$ are not B-rigid, either (refer to, e.g., [4], Example 1.1). Nonetheless, the aim of this paper is to show that B-rigidity is equivalent to C-rigidity in case of simple convex polytopes supporting quasitoric manifolds. This affirmatively answers a question in [4], Section 8, as follows.

Theorem 3. Let $P$ be a simple convex polytope supporting a quasitoric manifold, and let $K=(\partial P)^{*}$ be the dual of the boundary of $P$. Then $B$-rigidity of $K$ is equivalent to $C$-rigidity of $P$.

We organize this paper as follows. In Section 2, we will collect some basic facts about the Stanley-Reisner face ring $\mathbf{k}(K)$ and its Tor-algebra $\operatorname{Tor}_{\mathbf{k}[\mathbf{v}]}(\mathbf{k}(K), \mathbf{k})$ of $\mathbf{k}(K)$ and recall some well-known terminology used in this paper. In Section 3, we give a proof of our main Theorem 3.

\section{Stanley-Reisner Face Ring and Moment-Angle Complexes}

The aim of this section is to set up some notations and briefly collect some basic material necessary for the proof of main Theorem 3 given in Section 3. In particular, we recall wellknown facts about Stanley-Reisner face ring, following the works of $[5,6]$. Refer to $[1,4]$ for more details and other notations used in this paper.

To do so, let $\mathbf{k}[\mathbf{v}]=\mathbf{k}\left[v_{1}, v_{2}, \ldots, v_{m}\right]$ be the polynomial algebra over $\mathbf{k}$ on $m$ variables $v_{1}, v_{2}, \ldots, v_{m}$ of degree 2 . Let $\mathbb{N}$ denote the set of nonnegative integers and $\mathbf{a}=$ $\left(a_{1}, a_{2}, \ldots, a_{m}\right) \in \mathbb{N}^{m}$. Each monomial in $\mathbf{k}[\mathbf{v}]$ has the form $\mathbf{v}^{\mathbf{a}}=v_{1}^{a_{1}} v_{2}^{a_{2}} \cdots v_{m}^{a_{m}}$. Thus $\mathbf{k}[\mathbf{v}]$ is $\mathbb{N}^{m}$-graded, so that we have

$$
\mathbf{k}[\mathbf{v}]=\oplus_{\mathbf{a} \in \mathbb{N}^{m}} \mathbf{k}[\mathbf{v}]_{\mathbf{a}}, \quad \mathbf{k}[\mathbf{v}]_{\mathbf{a}} \cdot \mathbf{k}[\mathbf{v}]_{\mathbf{b}}=\mathbf{k}[\mathbf{v}]_{\mathbf{a}+\mathbf{b}},
$$

where $\mathbf{k}[\mathbf{v}]_{\mathbf{a}}$ denotes the vector space $\left\langle\mathbf{v}^{\mathbf{a}}\right\rangle$ over $\mathbf{k}$ spanned by $\mathbf{v}^{\mathbf{a}}$.
The Stanley-Reisner ideal $I_{K}$ of a simplicial complex $K$ is defined as

$$
I_{K}=\left\langle\mathbf{v}^{\tau} \mid \tau \notin K\right\rangle
$$

and its quotient $\operatorname{ring} \mathbf{k}(K)=\mathbf{k}[\mathbf{v}] / I_{K}$ is called the StanleyReisner face ring of $K$.

Since $\mathbf{k}(K)$ has finitely generated graded $\mathbf{k}[\mathbf{v}]$-module, there exists a free resolution of $\mathbf{k}(K)$ of length at most $m$. Moreover, it has an $\mathbb{N}^{m}$-graded minimal free resolution as follows:

$$
0 \longleftarrow \mathbf{k}(K) \stackrel{\phi_{0}}{\longleftarrow} F_{0} \stackrel{\phi_{1}}{\longleftarrow} F_{1} \stackrel{\phi_{2}}{\longleftarrow} \ldots \stackrel{\phi_{h-1}}{\longleftarrow} F_{h-1} \stackrel{\phi_{h}}{\longleftarrow} F_{h} \longleftarrow 0,
$$

where each homomorphism $\phi_{i}$ is an $\mathbb{N}^{m}$-graded degreepreserving homomorphism.

Now, applying the functor $\otimes_{\mathbf{k}[\mathbf{v}]} \mathbf{k}$ to the sequence (5), we can obtain the following chain complex of $\mathbb{N}^{m}$-graded $\mathbf{k}[\mathbf{v}]$-modules:

$$
\begin{aligned}
& 0 \longleftarrow F_{0} \otimes_{\mathbf{k}[\mathbf{v}]} \mathbf{k} \stackrel{\phi_{1}^{\prime}}{\longleftarrow} F_{1} \otimes_{\mathbf{k}[\mathbf{v}]} \mathbf{k} \\
& \stackrel{\phi_{2}^{\prime}}{\longleftarrow} \cdots \stackrel{\phi_{h-1}^{\prime}}{\longleftarrow} F_{h-1} \otimes_{\mathbf{k}[\mathbf{v}]} \mathbf{k} \stackrel{\phi_{h}^{\prime}}{\longleftarrow} F_{h} \otimes_{\mathbf{k}[\mathbf{v}]} \mathbf{k} \longleftarrow 0,
\end{aligned}
$$

where each homomorphism $\phi_{i}^{\prime}$ is given by $\phi_{i} \otimes \mathrm{Id}_{\mathbf{k}}$. Since the free resolution (5) is minimal, the differentials $\phi_{i}^{\prime}$ are actually zero homomorphisms. Hence the $i$ th homology module of the above chain complex, denoted $\operatorname{Tor}_{\mathbf{k}[\mathbf{v}]}^{i}(\mathbf{k}(K), \mathbf{k})$, is given by $F_{i} \otimes_{\mathbf{k}[\mathbf{v}]} \mathbf{k}$. In particular, we have

$$
\operatorname{dim}_{\mathbf{k}} \operatorname{Tor}_{\mathbf{k}[\mathbf{v}]}^{i}(\mathbf{k}(K), \mathbf{k})=\operatorname{rank}_{\mathbf{k}[\mathbf{v}]} F_{i} .
$$

With these notations, by definition we have

$$
\begin{gathered}
\beta_{i}(K)=\operatorname{dim}_{\mathbf{k}} \operatorname{Tor}_{\mathbf{k}[\mathbf{v}]}^{i}(\mathbf{k}(K), \mathbf{k}), \\
\beta_{i, 2 j}(K)=\operatorname{dim}_{\mathbf{k}} \operatorname{Tor}_{\mathbf{k}[\mathbf{v}]}^{i, 2 j}(\mathbf{k}(K), \mathbf{k}) .
\end{gathered}
$$

In case of a simple convex polytope $P$, the bigraded Betti number $\beta_{i, 2 j}(P)$ is defined to be the bigraded Betti number $\beta_{i, 2 j}(K)$ of the simplicial complex $K=(\partial P)^{*}$ that is dual to the boundary $\partial P$ of $P$.

Next, we recall the notion of a regular sequence of the Stanley-Reisner face ring $\mathbf{k}(K)$ (refer to, e.g., [2], Section $5)$. The Krull dimension of $\mathbf{k}(K)$ is defined to be the maximal number of algebraically independent elements of $\mathbf{k}(K)$. Suppose that the Krull dimension of $\mathbf{k}(K)$ is $n$. A sequence $\left(\lambda_{1}, \lambda_{2}, \ldots, \lambda_{n}\right)$ of homogeneous elements of $\mathbf{k}(K)$ is called a homogeneous system of parameters if the Krull dimension of $\mathbf{k}(K) /\left\langle\lambda_{1}, \lambda_{2}, \ldots, \lambda_{n}\right\rangle$ is zero. A homogeneous system of parameters $\left(\lambda_{1}, \lambda_{2}, \ldots, \lambda_{n}\right)$ of $\mathbf{k}(K)$ is called regular if $\lambda_{i+1}$ is not a zero divisor in $\mathbf{k}(K) /\left\langle\lambda_{1}, \lambda_{2}, \ldots, \lambda_{i}\right\rangle$. Equivalently, a sequence $\left(\lambda_{1}, \lambda_{2}, \ldots, \lambda_{n}\right)$ is regular if $\lambda_{1}, \lambda_{2}, \ldots, \lambda_{n}$ are algebraically independent and if $\mathbf{k}(K)$ is a finite dimensional free $\mathbf{k}\left[\lambda_{1}, \lambda_{2}, \ldots, \lambda_{n}\right]$-module.

Finally, we close this section with reviewing the construction of a moment-angle complex associated with an abstract 
simplicial complex on a vertex set. To do so, let $m$ be a positive integer and let us denote by $[m]$ the set $\{1,2, \ldots, m\}$. Let $K$ be an abstract simplicial complex on the vertex set $[m]$. For each simplex $\sigma \in K$, we set

$$
B_{\sigma}\left(D^{2}, S^{1}\right)=\prod_{i=1}^{m} A_{i},
$$

where $D^{2}=\{z \in \mathbb{C}|| z \mid \leq 1\}, S^{1}=\partial D^{2}$, and

$$
A_{i}= \begin{cases}D^{2}, & i \in \sigma, \\ S^{1}, & i \in[m] \backslash \sigma .\end{cases}
$$

Then the moment-angle complex $\mathscr{Z}_{K}$ on $K$ is defined to be a subspace of $\left(D^{2}\right)^{m}$, as follows:

$$
\mathscr{Z}_{K}:=\bigcup_{\sigma \in K} B_{\sigma}\left(D^{2}, S^{1}\right) \subset\left(D^{2}\right)^{m} .
$$

When $K=2^{[m]}$, it is easy to see that $\mathscr{Z}_{K}=\left(D^{2}\right)^{m}$. On the other hand, when $K=2^{[m]} \backslash\{[m]\}$, where $2^{[m]}$ denotes the power set of $[m]$, it can be easily shown that $\mathscr{Z}_{K}=S^{2 m-1}$ (refer to, e.g., [7], Example 2.4). Since $\left(D^{2}\right)^{m}$ as a subspace of $\mathbb{C}^{m}$ is invariant under the standard action of $T^{m}$ on $\mathbb{C}^{m}$ given by

$$
\begin{gathered}
\left(\left(g_{1}, g_{2}, \ldots, g_{m}\right),\left(z_{1}, z_{2}, \ldots, z_{m}\right)\right) \\
\longmapsto\left(g_{1} z_{1}, g_{2} z_{2}, \ldots, g_{m} z_{m}\right)
\end{gathered}
$$

$\left(D^{2}\right)^{m}$ inherits a natural $T^{m}$-action whose orbit space is the unit cube $I^{m}:=[0,1]^{m} \subset \mathbb{R}_{\geq 0}^{m}$. This $T^{m}$-action on $\left(D^{2}\right)^{m}$ then induces a canonical $T^{m}$-action on the moment-angle complex $\mathscr{Z}_{K}$. Refer to [1], Chapters 6 and 7 for more details on a moment-angle complex $\mathscr{Z}_{K}$.

\section{Proof of Theorem 3}

The aim of this section is to give a proof of Theorem 3. Before doing it, we should remark that there seems to exist some confusing point at the end of [4], Section 8, where the authors erroneously claim the proof that C-rigidity implies B-rigidity for simple convex polytopes supporting quasitoric manifolds.

We begin with the following lemma.

Lemma 4. Let $P$ be a simple convex polytope supporting a quasitoric manifold $M$, and let $K=(\partial P)^{*}$ be the dual of the boundary of $P$. If $K$ is $B$-rigid, then $P$ is $C$-rigid.

Proof. To prove it, suppose that $K$ is B-rigid. Let $P^{\prime}$ be another simple convex polytope supporting a quasitoric manifold $M^{\prime}$ such that

$$
H^{*}(M ; \mathbf{k}) \cong H^{*}\left(M^{\prime} ; \mathbf{k}\right) .
$$

As before, let $K^{\prime}=\left(\partial P^{\prime}\right)^{*}$ be the simplicial complex which is the dual of the boundary of the simple convex polytope $P^{\prime}$. Then it follows from [4], Lemma 3.7, and Proposition 3.8 that as a ring

$$
\operatorname{Tor}_{\mathbf{k}[\mathbf{v}]}(\mathbf{k}(K), \mathbf{k}) \cong \operatorname{Tor}_{\mathbf{k}[\mathbf{v}]}\left(\mathbf{k}\left(K^{\prime}\right), \mathbf{k}\right) .
$$

Since we have a ring isomorphism $H^{*}\left(\mathscr{E}_{K} ; \mathbf{k}\right) \cong \operatorname{Tor}(\mathbf{k}(K), \mathbf{k})$ for any simplicial complex $K$ by Buchstaber and Panov ([1], Theorem 7.6, and [8], Theorem 4.7), it follows from (14) that we have a ring isomorphism between $H^{*}\left(\mathscr{Z}_{K} ; \mathbf{k}\right)$ and $H^{*}\left(\mathscr{Z}_{K^{\prime}} ; \mathbf{k}\right)$. Thus $K$ is combinatorially equivalent to $K^{\prime}$ by the assumption that $K$ is B-rigid. This implies that $P$ is also combinatorially equivalent to $P^{\prime}$, which proves that $P$ is $\mathrm{C}$ rigid.

Next, we show the following lemma.

Lemma 5. Let $P$ (resp. $\left.P^{\prime}\right)$ be a simple convex polytope supporting a quasitoric manifold $M$ (resp., $M^{\prime}$ ), and let $K=$ $(\partial P)^{*}$ (resp., $K^{\prime}=\left(\partial P^{\prime}\right)^{*}$ ) be the dual of the boundary of $P$ (resp., $\left.P^{\prime}\right)$. Assume that as a ring

$$
H^{*}\left(\mathscr{Z}_{K} ; \mathbf{k}\right) \cong H^{*}\left(\mathscr{Z}_{K^{\prime}} ; \mathbf{k}\right) \text {. }
$$

Then two Stanley-Reisner face rings $\mathbf{k}(K)$ and $\mathbf{k}\left(K^{\prime}\right)$ are isomorphic to each other, as rings.

Proof. To prove it, note first that as a ring

$$
\operatorname{Tor}_{\mathbf{k}[\mathbf{v}]}(\mathbf{k}(K), \mathbf{k}) \cong \operatorname{Tor}_{\mathbf{k}[\mathbf{v}]}\left(\mathbf{k}\left(K^{\prime}\right), \mathbf{k}\right) .
$$

In particular, we have

$$
\beta_{i, 2 j}(K)=\beta_{i, 2 j}\left(K^{\prime}\right)
$$

for all $i$ and $j$.

Next, we claim that the Stanley-Reisner face rings $\mathbf{k}(K)$ and $\mathbf{k}\left(K^{\prime}\right)$ are isomorphic to each other as rings. To prove it, let $m$ (resp. $m^{\prime}$ ) denote the number of facets of $P$ (resp., $P^{\prime}$ ). Since $\beta_{1,2}(K)$ (resp., $\beta_{1,2}\left(K^{\prime}\right)$ ) is equal to the number of facets of $P$ (resp. $P^{\prime}$ ) by the nice formula for bigraded Betti numbers by Hochster ([9], Theorem 5.2 or [4], Theorem 3.3), it follows from (17) that $m$ should be equal to $m^{\prime}$. Then, as in Section 2, consider an $\mathbb{N}^{m}$-graded minimal free resolution for $\mathbf{k}(K)$ as follows:

$$
0 \longleftarrow \mathbf{k}(K) \stackrel{\phi_{0}}{\longleftarrow} F_{0} \stackrel{\phi_{1}}{\longleftarrow} F_{1} \stackrel{\phi_{2}}{\longleftarrow} \ldots \stackrel{\phi_{h-1}}{\longleftarrow} F_{h-1} \stackrel{\phi_{h}}{\longleftarrow} F_{h} \longleftarrow 0,
$$

where each homomorphism $\phi_{i}$ is an $\mathbb{N}^{m}$-graded degreepreserving homomorphism. Similarly, let

$$
\begin{aligned}
& 0 \longleftarrow \mathbf{k}\left(K^{\prime}\right) \stackrel{\phi_{0}^{\prime}}{\longleftarrow} F_{0}^{\prime} \stackrel{\phi_{1}^{\prime}}{\longleftarrow} F_{1}^{\prime} \\
& \stackrel{\phi_{2}^{\prime}}{\longleftarrow} \ldots \stackrel{\phi_{h^{\prime}-1}^{\prime}}{\longleftarrow} F_{h^{\prime}-1}^{\prime} \stackrel{\phi_{h^{\prime}}^{\prime}}{\longleftarrow} F_{h^{\prime}}^{\prime} \longleftarrow 0
\end{aligned}
$$

be an $\mathbb{N}^{m}$-graded minimal resolution of $\mathbf{k}\left(K^{\prime}\right)$. Since the free resolution of $\mathbf{k}(K)$ is minimal, for each $i=1,2, \ldots, h$ we have

$$
\begin{aligned}
\operatorname{rank}_{\mathbf{k}[\mathbf{v}]}\left(F_{i}\right) & =\sum_{j} \beta_{i, 2 j}(K)=\sum_{j} \beta_{i, 2 j}\left(K^{\prime}\right) \\
& =\operatorname{rank}_{\mathbf{k}[\mathbf{v}]}\left(F_{i}^{\prime}\right) .
\end{aligned}
$$

In particular, this implies that $h$ is equal to $h^{\prime}$.

Now, we show that there are $\mathbb{N}^{m}$-graded degreepreserving isomorphisms $\psi_{i}(0 \leq i \leq h)$ from $F_{i}$ to $F_{i}^{\prime}$ so that the diagram (21) below commutes as follows: 


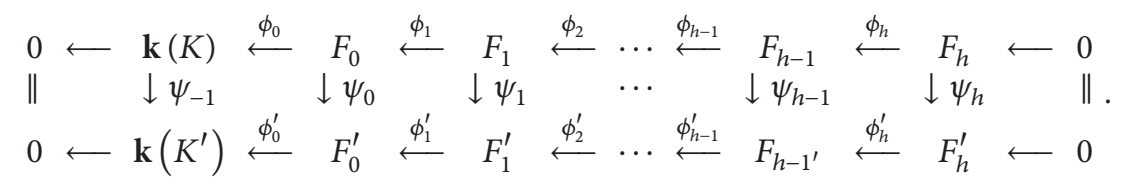

To do so, recall first that by (16) there is a ring isomorphism $\kappa_{i}$ from $\operatorname{Tor}_{\mathbf{k}[\mathbf{v}]}^{i}(\mathbf{k}(K), \mathbf{k})$ to $\operatorname{Tor}_{\mathbf{k}[\mathbf{v}]}^{i}\left(\mathbf{k}\left(K^{\prime}\right), \mathbf{k}\right)$ for each $i$. Hence, in particular, $\kappa_{i}$ induces an isomorphism from $\operatorname{Tor}_{\mathbf{k}[\mathbf{v}]}^{i, 2}(\mathbf{k}(K), \mathbf{k})$ to $\operatorname{Tor}_{\mathbf{k}[\mathbf{v}]}^{i, 2}\left(\mathbf{k}\left(K^{\prime}\right), \mathbf{k}\right)$ for each $i$. On the other hand, since $\kappa_{i}$ is a ring isomorphism and $\beta_{i, 2 j}(K)$ is equal to $\beta_{i, 2 j}\left(K^{\prime}\right)$ for all $i$ and $j$, this implies that actually $\kappa_{i}$ should be an $\mathbb{N}^{m}$-graded degree-preserving isomorphism from $\operatorname{Tor}_{\mathbf{k}[\mathbf{v}]}^{i}(\mathbf{k}(K), \mathbf{k})$ to $\operatorname{Tor}_{\mathbf{k}[\mathbf{v}]}^{i}\left(\mathbf{k}\left(K^{\prime}\right), \mathbf{k}\right)$ for each $i$. As noted in Section 2, recall also that there are $\mathbb{N}^{m}$-graded degreepreserving isomorphisms $\eta_{i}$ and $\eta_{i}^{\prime}$ such that

$$
\begin{gathered}
\eta_{i}: \operatorname{Tor}_{\mathbf{k}[\mathbf{v}]}^{i}(\mathbf{k}(K), \mathbf{k}) \longrightarrow F_{i} \otimes_{\mathbf{k}[\mathbf{v}]} \mathbf{k} \longrightarrow F_{i}, \\
\eta_{i}^{\prime}: \operatorname{Tor}_{\mathbf{k}[\mathbf{v}]}^{i}\left(\mathbf{k}\left(K^{\prime}\right), \mathbf{k}\right) \longrightarrow F_{i}^{\prime} \otimes_{\mathbf{k}[\mathbf{v}]} \mathbf{k} \longrightarrow F_{i}^{\prime} .
\end{gathered}
$$

So it is now easy to see that $\psi_{i}=\eta_{i}^{\prime} \circ \kappa_{i} \circ \eta_{i}^{-1}$ is an $\mathbb{N}^{m}$ graded degree-preserving isomorphism from $F_{i}$ to $F_{i}^{\prime}$ which automatically makes the diagram (21) commute, as desired.

Moreover, by the standard argument using the diagramchasing we can also construct an $\mathbb{N}^{m}$-graded degreepreserving homomorphism $\psi_{-1}$ between $\mathbf{k}(K)$ and $\mathbf{k}\left(K^{\prime}\right)$ in such a way that the diagram (21) commutes. To be more precise, let $x \in \mathbf{k}(K)$. Then there is an element $y \in F_{0}$ such that $\phi_{0}(y)=x$. We then define $\psi_{-1}: \mathbf{k}(K) \rightarrow \mathbf{k}\left(K^{\prime}\right)$ by $\psi_{-1}(x)=\phi_{0}^{\prime} \circ \psi_{0}(y)$. Then it is well defined; that is, this definition is independent of the choice of $y$. Indeed, let $y^{\prime} \in F_{0}$ such that $\phi_{0}\left(y^{\prime}\right)=x$. Then, since $\phi_{0}\left(y-y^{\prime}\right)=0$, there is an element $z \in F_{1}$ such that $y-y^{\prime}=\phi_{1}(z)$. Thus we have

$$
\begin{aligned}
\phi_{0}^{\prime} \circ \psi_{0}(y)-\phi_{0}^{\prime} \circ \psi_{0}\left(y^{\prime}\right) & =\phi_{0}^{\prime} \circ \psi_{0}\left(y-y^{\prime}\right) \\
& =\left(\phi_{0}^{\prime} \circ \phi_{1}^{\prime}\right) \circ \psi_{1}(z)=0,
\end{aligned}
$$

as desired.

Finally, it is easy to see that by using the five-lemma ([10], p. 169) the ring homomorphism $\psi_{-1}$ is also an $\mathbb{N}^{m}$ graded degree-preserving ring isomorphism between $\mathbf{k}(K)$ and $\mathbf{k}\left(K^{\prime}\right)$. This, in particular, completes the proof of Lemma 5.

Recall now that the Stanley-Reisner face ring $\mathbf{k}(K)$ is given by $\mathbf{k}[\mathbf{v}] / I_{K}$, where $I_{K}$ denotes the Stanley-Reisner ideal defined as $\left\langle\mathbf{v}^{\tau} \mid \tau \notin K\right\rangle$. Let $J$ be the ideal of $\mathbf{k}(K)$ generated by a regular sequence of homogeneous system of parameters of degree 2 elements. Then the cohomology $\operatorname{ring} H^{*}(M ; \mathbf{k})$ is isomorphic to

$$
\frac{\mathbf{k}(K)}{J} \text {. }
$$

Then we need the following lemma ([1], Lemma 3.35, or [4], Lemma 3.6).
Lemma 6. Let $J$ be an ideal generated by a regular sequence of $\mathbf{k}(K)$. Then we have the following algebra isomorphism:

$$
\operatorname{Tor}_{\mathbf{k}[\mathbf{v}]}(\mathbf{k}(K), \mathbf{k}) \cong \operatorname{Tor}_{\mathbf{k}[\mathbf{v}] / J}\left(\frac{\mathbf{k}(K)}{J}, \mathbf{k}\right)
$$

The following lemma will also play an important role in the proof of Theorem 3 .

Lemma 7. Under the same assumptions as in Lemma 5, two rings $\mathbf{k}(K) / J$ and $\mathbf{k}\left(K^{\prime}\right) / J^{\prime}$ are isomorphic to each other.

Proof. By Lemmas 5 and 6, we have an algebra isomorphism as follows:

$$
\operatorname{Tor}_{\mathbf{k}[\mathbf{v}] / J}\left(\frac{\mathbf{k}(K)}{J}, \mathbf{k}\right) \cong \operatorname{Tor}_{\mathbf{k}[\mathbf{v}] / J}\left(\frac{\mathbf{k}\left(K^{\prime}\right)}{J^{\prime}}, \mathbf{k}\right)
$$

By applying the same arguments as in the proof of Lemma 5 to $\mathbb{N}^{m}$-graded minimal free resolutions of $\mathbf{k}(K) / J$ and $\mathbf{k}\left(K^{\prime}\right) / J^{\prime}$ derived from the isomorphism (26) as in (16), it is now straightforward to see that two rings $\mathbf{k}(K) / J$ and $\mathbf{k}\left(K^{\prime}\right) / J^{\prime}$ are isomorphic to each other, as desired.

Finally, we are ready to prove our main theorem of this section, as follows.

Theorem 8. Let $P$ be a simple convex polytope supporting a quasitoric manifold $M$, and let $K=(\partial P)^{*}$ be the dual of the boundary of $P$. If $P$ is $C$-rigid, then $K$ is $B$-rigid.

Proof. To prove it, as before let $P$ (resp., $P^{\prime}$ ) be a simple convex polytope supporting a quasitoric manifold $M$ (resp. $M^{\prime}$ ), and let $K=(\partial P)^{*}$ (resp., $K^{\prime}=\left(\partial P^{\prime}\right)^{*}$ ) be the dual of the boundary of $P$ (resp., $P^{\prime}$ ). Assume further that as a ring

$$
H^{*}\left(\mathscr{Z}_{K} ; \mathbf{k}\right) \cong H^{*}\left(\mathscr{Z}_{K^{\prime}} ; \mathbf{k}\right) .
$$

By Lemma 7, we then have a ring isomorphism

$$
H^{*}(M ; \mathbf{k}) \cong \frac{\mathbf{k}(K)}{J} \cong \frac{\mathbf{k}\left(K^{\prime}\right)}{J^{\prime}} \cong H^{*}\left(M^{\prime} ; \mathbf{k}\right) \text {. }
$$

Since $M$ (resp., $M^{\prime}$ ) is a quasitoric manifold over a simple convex polytope (resp., $P^{\prime}$ ), it follows from the assumption of $P$ being C-rigid that $P$ is combinatorially equivalent to $P^{\prime}$. So $K$ should be also combinatorial equivalent to $K^{\prime}$, completing the proof of Theorem 8 .

Proof of Theorem 3. The proof of Theorem 3 now follows immediately from Lemma 4 and Theorem 8 . 


\section{Conflict of Interests}

The author declares that there is no conflict of interests regarding the publication of this paper.

\section{Acknowledgments}

The author is grateful to the anonymous reader for valuable comments on this paper. This work was supported by the National Research Foundation of Korea (NRF) Grant funded by the Korea Government (MSIP) (no. 2014001824).

\section{References}

[1] V. M. Buchstaber and T. E. Panov, Torus Actions and Their Applications in Topology and Combinatorics, vol. 24 of University Lecture Series, American Mathematical Society, Providence, RI, USA, 2002.

[2] M. W. Davis and T. Januszkiewicz, "Convex polytopes, Coxeter orbifolds and torus actions," Duke Mathematical Journal, vol. 62, no. 2, pp. 417-451, 1991.

[3] V. Buchstaber, "Lectures on toric topology, lecture note based on lectures at KAIST," Trends in Mathematics, vol. 10, pp. 1-64, 2008.

[4] S. Choi, T. Panov, and D. Y. Suh, "Toric cohomological rigidity of simple convex polytopes," Journal of the London Mathematical Society, vol. 82, no. 2, pp. 343-360, 2010.

[5] X. Cao and Z. Lü, "Möbius transform, moment-angle complexes and Halperin-Carlsson conjecture," Journal of Algebraic Combinatorics, vol. 35, no. 1, pp. 121-140, 2012.

[6] E. Miller and B. Sturmfels, Combinatorial Commutative Algebra, vol. 227 of Graduate Texts in Mathematics, Springer, Berlin, Germany, 2005.

[7] A. Bahri, M. Bendersky, F. R. Cohen, and S. Gitler, "The polyhedral product functor: a method of decomposition for moment-angle complexes, arrangements and related spaces," Advances in Mathematics, vol. 225, no. 3, pp. 1634-1668, 2010.

[8] T. Panov, "Cohomology of face rings, and torus actions," in Surveys in Contemporary Mathematics, vol. 347 of London Mathematical Society Lecture Note Series, pp. 165-201, Cambridge University Press, Cambridge, UK, 2008.

[9] M. Hochster, "Cohen-Macaulay rings, combinatorics, and simplicial complexes," in Proceedings of the 2nd Oklahoma Conference, pp. 171-223, Dekker, New York, NY, USA, 1977.

[10] S. Lang, Algebra, Addison-Wesley, New York, NY, USA, 3rd edition, 1999. 


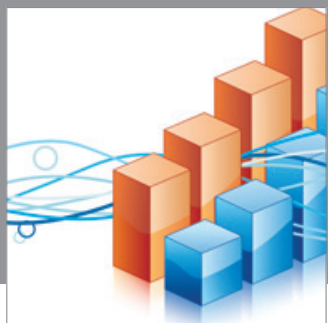

Advances in

Operations Research

mansans

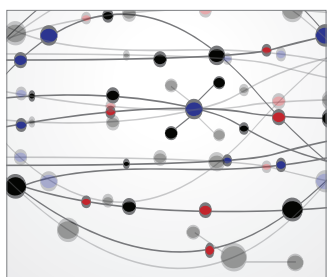

The Scientific World Journal
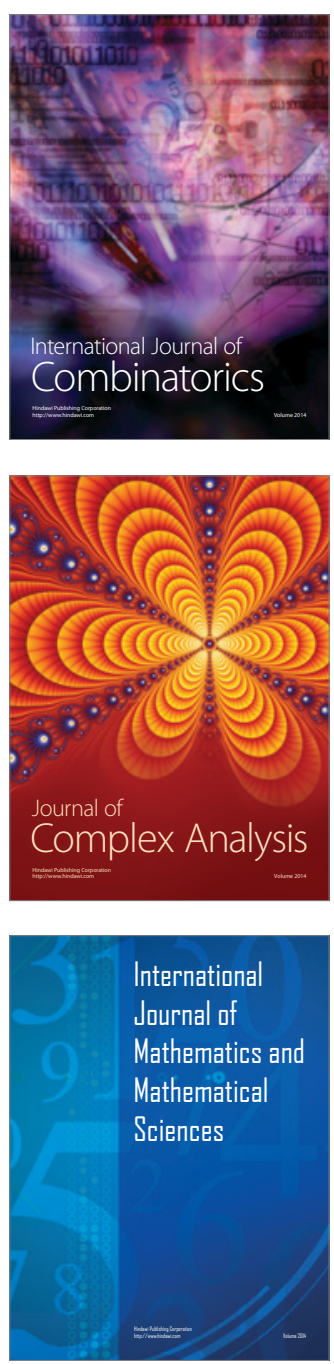
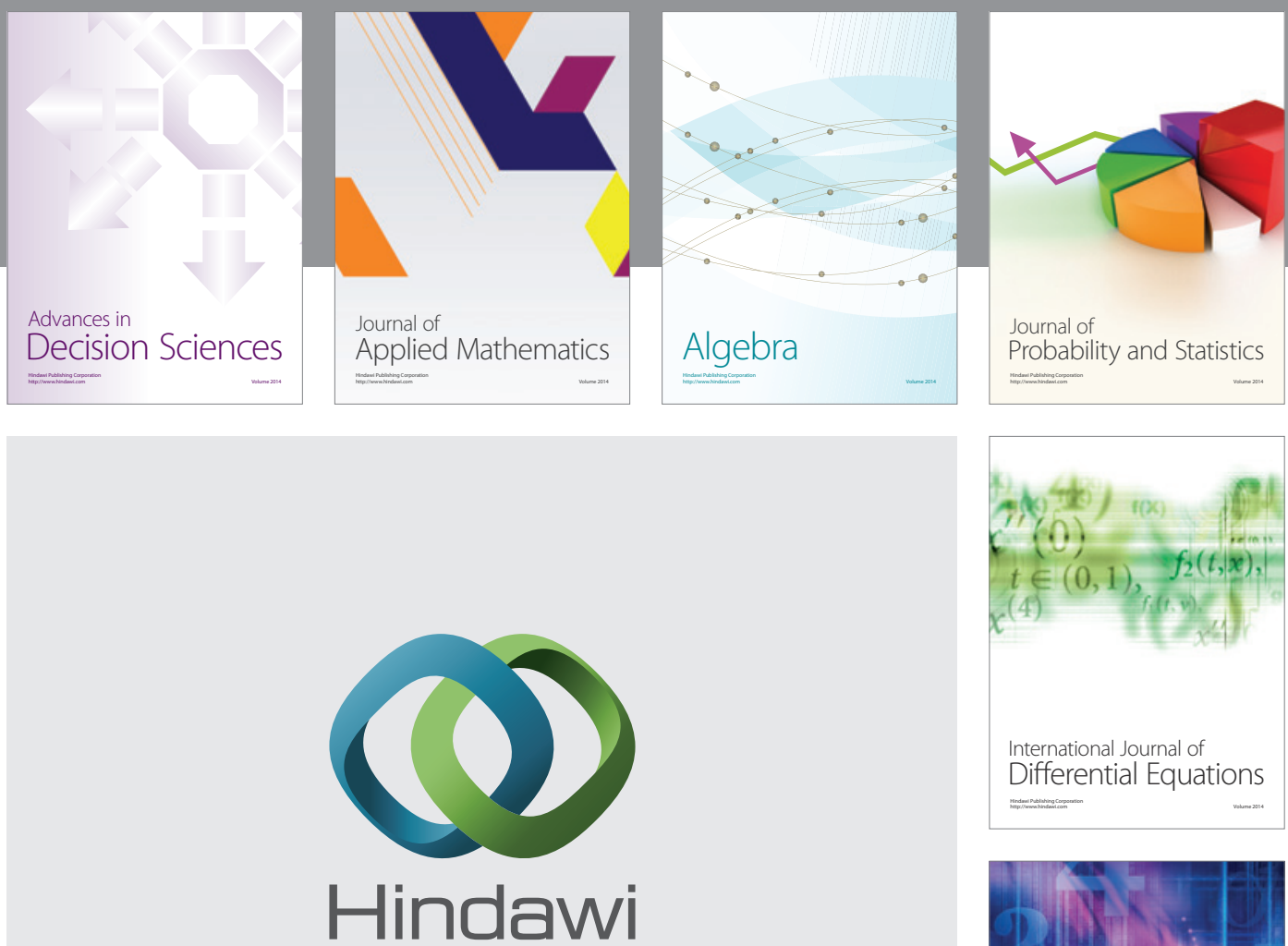

Submit your manuscripts at http://www.hindawi.com
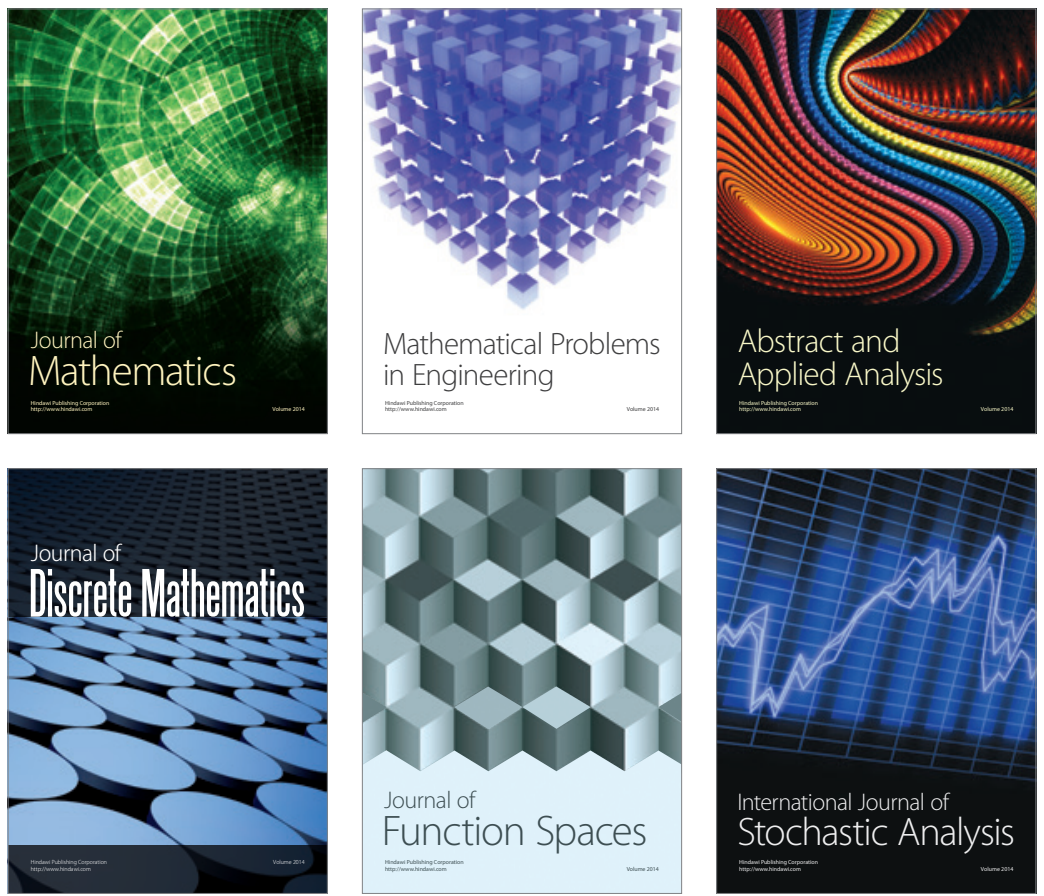

Journal of

Function Spaces

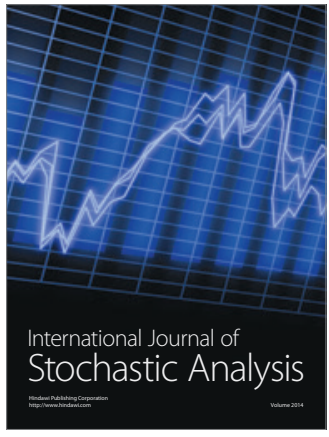

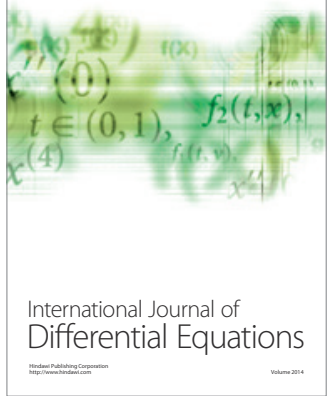
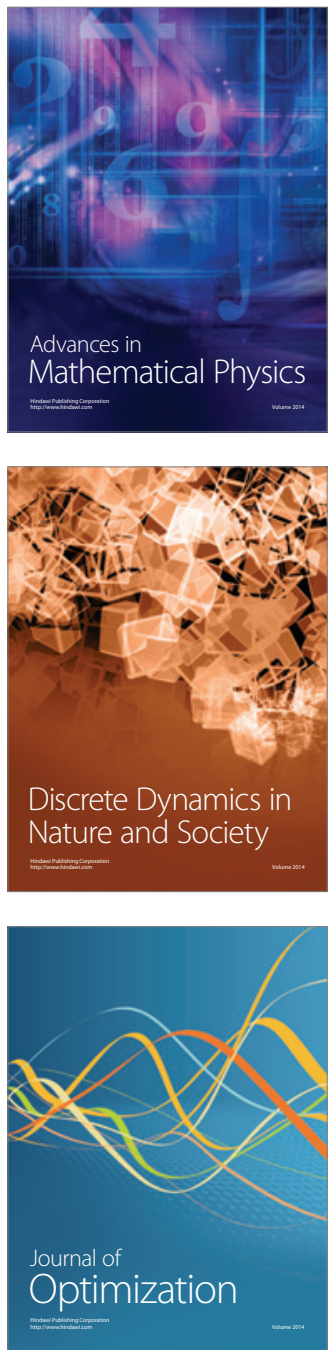